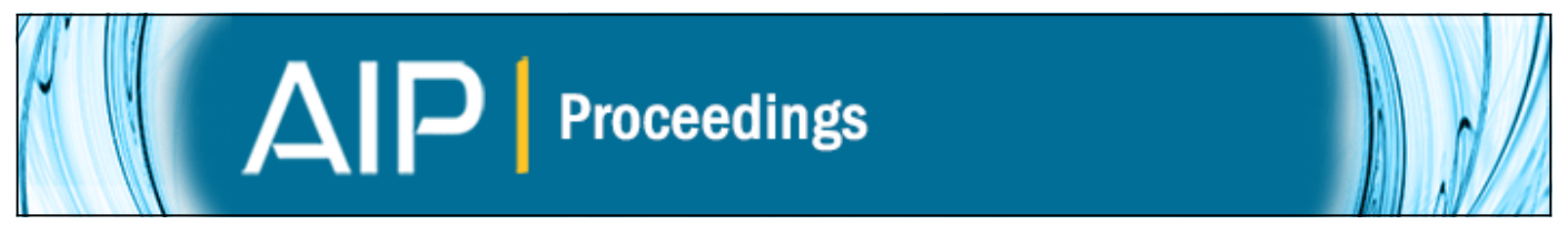

\title{
Cosmic ray source abundances and the acceleration of cosmic rays
}

J. S. George, M. E. Wiedenbeck, A. F. Barghouty, W. R. Binns, E. R. Christian, A. C. Cummings, P. L. Hink, J. Klarmann, R. A. Leske, M. Lijowski, R. A. Mewaldt, E. C. Stone, T. T. von Rosenvinge, and N. E. Yanasak

Citation: AIP Conference Proceedings 528, 437 (2000); doi: 10.1063/1.1324355

View online: http://dx.doi.org/10.1063/1.1324355

View Table of Contents: http://scitation.aip.org/content/aip/proceeding/aipcp/528?ver=pdfcov

Published by the AIP Publishing 


\title{
Cosmic Ray Source Abundances and the Acceleration of Cosmic Rays
}

\author{
J. S. George ${ }^{1}$, M. E. Wiedenbeck ${ }^{4}$, A. F. Barghouty ${ }^{1}$, W. R. Binns ${ }^{2}$, E. R. Christian ${ }^{3}$, \\ A. C. Cummings ${ }^{1}$, P. L. Hink ${ }^{2}$, J. Klarmann ${ }^{2}$, R. A. Leske ${ }^{1}$, M. Lijowski ${ }^{2}$, \\ R. A. Mewaldt ${ }^{1}$, E. C. Stone ${ }^{1}$, T. T. von Rosenvinge ${ }^{3}$, N. E. Yanasak ${ }^{4}$ \\ ${ }^{1}$ California Institute of Technology, ${ }^{2}$ Washington University, ${ }^{3}$ NASA / Goddard Space Flight Center, \\ ${ }^{4}$ Jet Propulsion Laboratory
}

\begin{abstract}
The galactic cosmic ray elemental source abundances display a fractionation that is possibly based on first ionization potential (FIP) or volatility. A few elements break the general correlation of FIP and volatility and the abundances of these may help to distinguish between models for the origin of the cosmic ray source material. Data from the Cosmic Ray Isotope Spectrometer instrument on NASA's Advanced Composition Explorer spacecraft were used to derive source abundances for several of these elements ( $\mathrm{Na}, \mathrm{Cu}, \mathrm{Zn}, \mathrm{Ga}, \mathrm{Ge}$ ). Three ( $\mathrm{Na}, \mathrm{Cu}, \mathrm{Ge}$ ) show depletions which could be consistent with a volatility-based source fractionation model.
\end{abstract}

\section{INTRODUCTION}

The elemental abundances of the galactic cosmic rays (GCRs) reflect the abundances at their source and their evolution during propagation through the Galaxy. Careful modeling of the transport of cosmic rays through the intervening matter can provide an estimate of the source composition. The GCR source material is found to be similar in composition to the pool of material from which the solar system was formed, but with an observed elemental fractionation based on chemical properties such as the first ionization potential (FIP) or volatility (as indicated, for example, by the condensation temperature). The Cosmic Ray Isotope Spectrometer (1) (CRIS) on NASA's Advanced Composition Explorer (ACE) spacecraft has been measuring cosmic ray abundances for elements between helium $(Z=2)$ and selenium $(Z=34)$ at energies between 50 and $600 \mathrm{MeV} /$ nucleon for over two years. These new data may help to determine what physical parameter controls the fractionation and provide a vital clue for understanding the source of galactic cosmic rays and the acceleration mechanisms that power them.

There are several explanations for the observed depletion of certain elements in the galactic cosmic ray source. One comes from a similar observation in the abundances of solar energetic particles, coronal material, and the solar wind. In those cases the elemental fractionation is ordered by the first ionization potential (FIP), or possibly first ionization time (FIT) (see e.g. (2)). Apparently, elements with a lower FIP (or FIT) are more easily ion- ized, enabling them to be more easily transported from the photosphere into the corona (3). The similarity of this depletion pattern to that seen in the GCRs led Cassé et al. (4), Meyer (5), and others to speculate that the GCR seed population might be stellar coronal material. An alternative explanation for the GCR fractionation is that the cosmic rays are grain destruction products whose relative abundances are controlled by their condensation temperatures, or volatility $(6,7)$. The possibility that the GCR source material comes from dust grains in the interstellar medium has recently been explored again in detail (8).

The two explanations for the GCR fractionation lead to very different views of the cosmic ray source. Fortunately, the general correlation between FIP and volatility is not complete; the abundances of several elements might be used to distinguish between them as an ordering parameter. Four such elements, copper, zinc, gallium, and germanium, lie just beyond the iron-nickel peak where abundances fall rapidly with increasing nuclear charge and secondary contributions from fragmentation of heavier nuclei are minimal. A fifth key element, sodium, is many times more abundant than zinc, but contains an estimated $70 \%$ contribution from secondary fragments, notably from magnesium and silicon. The sodium source abundance is much more sensitive to errors in the fragmentation cross-sections and the total amount of interstellar material traversed than the trans-nickel elements for which source abundances can be more reliably determined. For this reason, different methods were used 
here to estimate the source abundances of sodium and the heavier elements.

\section{TRANS-NICKEL ELEMENTS}

Figure 1 shows a charge histogram of selected heavy element data recorded by CRIS over a two year period. The sub-peaks in copper and zinc correspond to different isotopes which were resolved for the first time in the CRIS instrument. The relative abundances of these isotopes, with relatively large statistical uncertainties, are consistent with the corresponding solar system values.

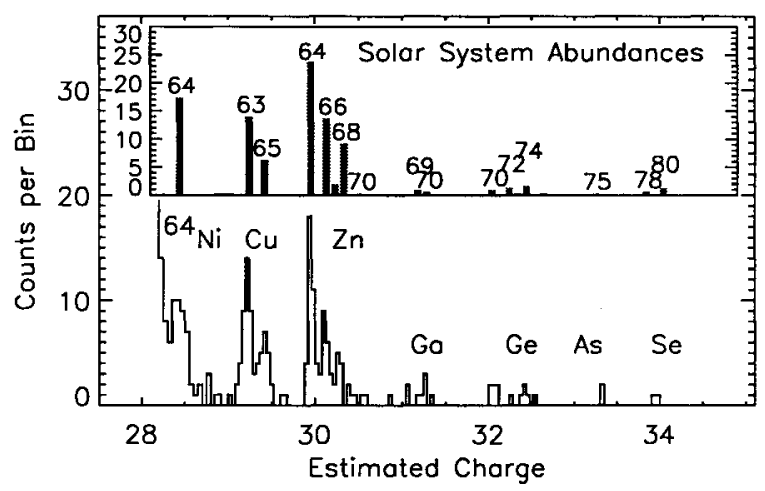

FIGURE 1. CRIS charge histogram for trans-nickel elements compared with solar system abundances (9).

A leaky box propagation model developed at the Naval Research Laboratory for other types of propagation studies (10) was used to model the effects of cosmic ray transport through the Galaxy. All stable isotopes (and those unstable on cosmic ray time scales) from $Z=21$ to $Z=41$ were propagated by solving the leaky box equations in the "weighted-slab" approximation in which nuclei are followed as they pass through successive thin slabs of interstellar material. The interstellar medium was taken to be $90 \%$ hydrogen and $10 \%$ helium by number. Losses due to fragmentation, radioactive decay, and escape from the Galaxy were taken into account, as well as gains from spallation and radioactive decay of heavier nuclides. The fragmentation of elements above zirconium $(Z=40)$ make negligible contributions to the copper-germanium ( $Z=29$ 32) region.

The model used the partial cross-sections of Silberberg $\&$ Tsao $(11,12)$. The escape mean free path, $\Lambda_{\mathrm{esc}}$, was taken to be a function of rigidity, $R$, and particle velocity, $\beta$, and gave results consistent with CRIS observations for the sub-iron/iron ratio.

$$
\begin{aligned}
& \Lambda_{\text {esc }}=17.2 \beta \quad \mathrm{g} / \mathrm{cm}^{2}, \quad R<4.0 \mathrm{GV} \\
& \Lambda_{\mathrm{esc}}=17.2 \beta(R / 4.0 \mathrm{GV})^{-0.6}, \quad R>4.0 \mathrm{GV}
\end{aligned}
$$

The source abundances were taken initially to be those of the solar system (9) with elemental abundances adjusted as necessary to fit the observed values. Figure 2 shows the relative abundances of 20 elements near Earth. The calculated abundances include the effects of solar modulation with a modulation parameter $\phi=500 \mathrm{MV}$.

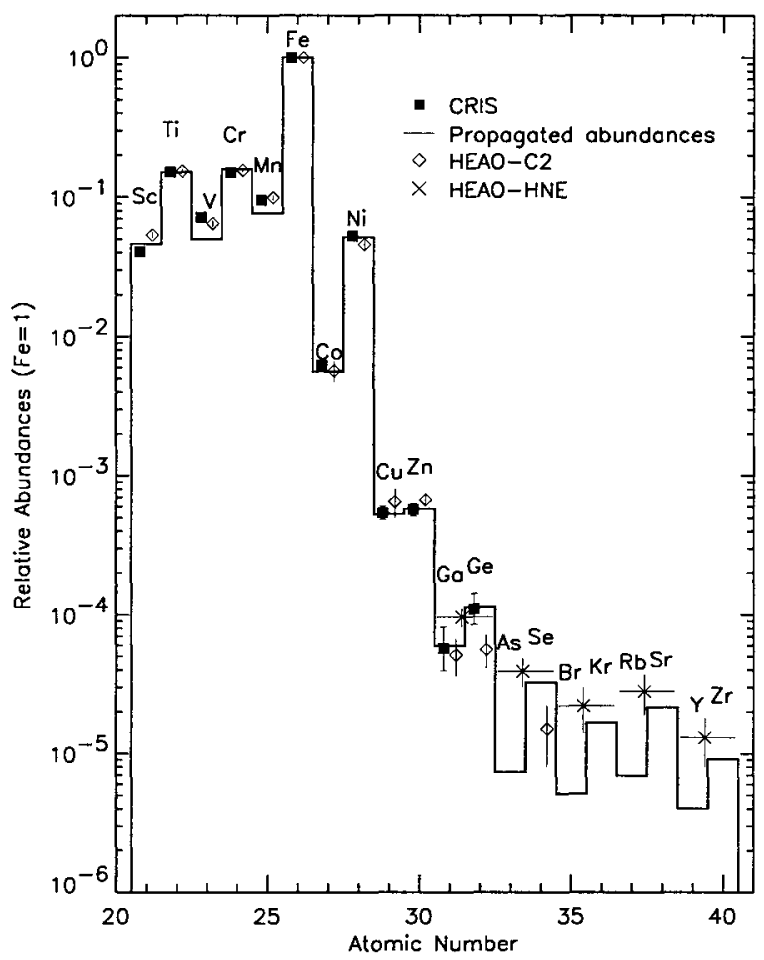

FIGURE 2. Relative near-Earth elemental abundances ( $\mathrm{Fe} \equiv 1)$. The histogram shows the results of the propagation calculation compared with HEAO-C2 data (13) and HEAO-HNE "lowenergy" charge pair measurements (14).

The propagation model accounts reasonably well for the sub-iron elements $(21 \leq \mathrm{Z} \leq 25)$. These elements have minimal source abundances and are almost purely secondary. The discrepancies for vanadium and manganese are comparable to the uncertainties in the fragmentation cross-sections. Source abundances of elements heavier than iron were adjusted so that the propagated abundances matched CRIS data where available. Above germanium, source values were adjusted in pairs so that the sum of the propagated odd and even charge elements matched the measurements by Binns et al. (14). With the calculated abundances in agreement with observations above and below the elements of interest, secondary contributions to the copper-germanium region should be well represented.

The ratios of the galactic cosmic ray source (GCRS) abundances to the corresponding solar system (SS) values (9) are summarized in Table 1 for the trans-nickel elements. The uncertainties in the GCRS/SS ratios re- 
flect the $1 \sigma$ statistical uncertainties in the calculated GCR sources and solar system abundances, as well as an estimated uncertainty in the fragmentation cross-sections. The secondary contributions for copper, zinc, gallium, and germanium were calculated to be $16 \%, 6 \%, 23 \%$, and $11 \%$, respectively, by repeating the propagation calculation with one element at a time removed from the source. A $20 \%$ overall uncertainty in the cross-sections contributed at most a $5 \%$ uncertainty to the calculated source abundance.

Table 1. GCR Source relative to solar system $\left(\mathrm{Fe} \equiv 10^{6}\right)$.

\begin{tabular}{lccc}
\hline Element & GCRS $^{*}$ & SS $^{\dagger}$ & GCRS/SS \\
\hline Copper & $463 \pm 49$ & $580 \pm 64$ & $0.80 \pm 0.12$ \\
Zinc & $573 \pm 58$ & $1400 \pm 62$ & $0.41 \pm 0.05$ \\
Gallium & $45 \pm 14$ & $42 \pm 2.9$ & $1.07 \pm 0.34$ \\
Germanium & $90 \pm 21$ & $132 \pm 13$ & $0.68 \pm 0.17$ \\
\hline
\end{tabular}

* This work.

+ Anders \& Grevesse (9).

\section{SODIUM SOURCE ABUNDANCE}

To determine the source abundance of sodium, several "purely secondary" nuclides with similar masses were used to constrain the calculated secondary corrections. In particular, the observed abundances of ${ }^{21} \mathrm{Ne},{ }^{19} \mathrm{~F}$, and ${ }^{17} \mathrm{O}$ should contain fragmentation products from the same parents that produce sodium. A series of steady-state leaky box propagation calculations (model described in (15)) were carried out while varying the escape mean free path, $\Lambda_{\text {esc }}$. For each $\Lambda_{\text {esc }}$ the source ratios of ${ }^{20} \mathrm{Ne},{ }^{22} \mathrm{Ne}$, ${ }^{23} \mathrm{Na},{ }^{25} \mathrm{Mg},{ }^{26} \mathrm{Mg},{ }^{27} \mathrm{Al},{ }^{28} \mathrm{Si},{ }^{29} \mathrm{Si}$, and ${ }^{30} \mathrm{Si}$ relative to the stable primary isotope ${ }^{24} \mathrm{Mg}$ were adjusted to reproduce the locally observed values. Source abundances of heavier nuclides were held fixed at solar system values modified by a FIP fractionation. The source abundances of ${ }^{17} \mathrm{O},{ }^{19} \mathrm{~F}$, and ${ }^{21} \mathrm{Ne}$ were also based on solar system values, although these are negligible compared to the secondaries produced during propagation.

For each value of $\Lambda_{\mathrm{esc}}$, the sodium source abundance needed to account for the observed sodium is determined, as well as the relative difference between the calculated and observed values of the three secondary nuclides being used to constrain the model. Figure 3 shows the correlation between the calculated sodium source abundance and these relative differences. When each secondary isotope is taken individually, our best estimate of the sodium source abundance corresponds to the point on the curve where the calculated and observed abundances of the tracer isotope agree (" $0 \%$ difference"). These val-

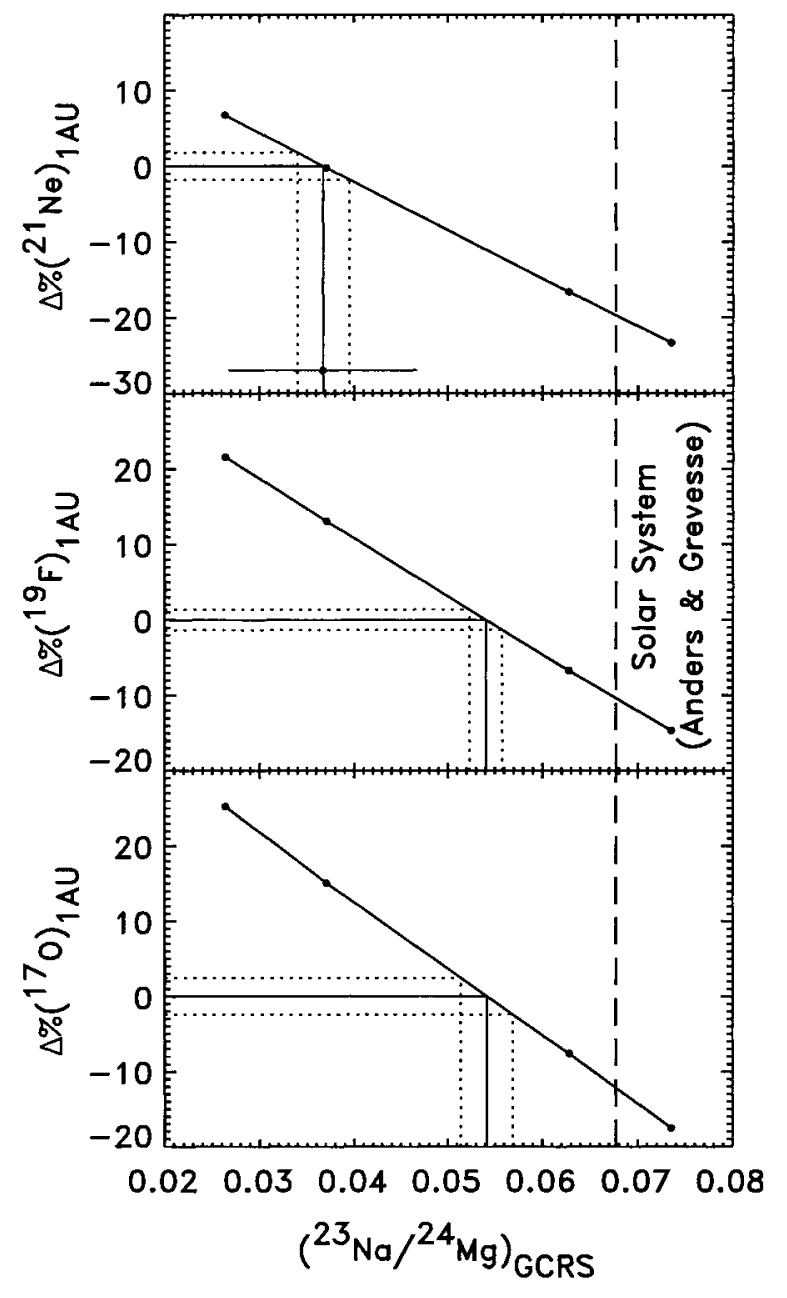

FIGURE 3. Constraints on the ${ }^{23} \mathrm{Na} /{ }^{24} \mathrm{Mg}$ source ratio. The vertical axis represents the percent difference $(\Delta \%)$ between the calculated and observed abundances of nearby secondary isotopes near Earth. The point in the top panel illustrates the statistical uncertainty in the calculated ${ }^{23} \mathrm{Na} /{ }^{24} \mathrm{Mg}$ source ratio due to uncertainty in the local measurements. The solar system value (9) is indicated by the vertical dashed line.

ues are indicated by the light solid lines in the figure, and the $1 \sigma$ statistical uncertainty limits are indicated as dotted lines. The differences between the three determinations of the sodium source abundance could be due, for example, to errors in the cross sections for producing the various secondaries.

A weighted average of the three determinations of the sodium source abundance was calculated and the spread among the different values used as an indication of the uncertainty arising from the calculation of the correction for secondary ${ }^{23} \mathrm{Na}$. The statistical uncertainty in the measurement of sodium was included to obtain a best estimate for the source ratio ${ }^{23} \mathrm{Na} /{ }^{24} \mathrm{Mg}$ of $0.048 \pm 0.015$. 
Compared to the solar system (9) ratio of 0.067 , sodium was found to be marginally depleted in the GCR source relative to other elements with similar first ionization potential. The isotopic ratio was normalized to elemental iron (Fe $\equiv 1$ ) using the HEAO (13) $\mathrm{Mg} / \mathrm{Fe}$ GCRS value of $1.03 \pm 0.03$ and the solar system isotopic and elemental abundances (9), resulting in a source GCRS/SS ratio for $\mathrm{Na} / \mathrm{Fe}$ of $0.62 \pm 0.19$.

\section{DISCUSSION \& SUMMARY}

Figure 4 shows the GCRS/SS ratio for all five elements studied here as a function of the first ionization potential. HEAO data (13) are added to provide a context for the CRIS measurements and one possible FIP parameterization is drawn as a solid line. Gallium and zinc are consistent with FIP as the ordering parameter. With only ten events, the gallium measurement is also consistent with volatility models. Zinc, in spite of the good statistical accuracy, does not discriminate between models well because it is in the intermediate FIP region where some depletion is likely. Copper and germanium show respective depletions of $1.7 \sigma$ and $1.9 \sigma$ relative to iron, consistent with what might be expected if volatility were the relevant parameter. The apparent depletions of the refractory elements magnesium and silicon can be interpreted as mass effects in the volatility model (8) and do not necessarily reduce the significance of the copper and germanium results.

The CRIS instrument is providing new measurements of key elemental source abundances in the galactic cosmic rays. Two of these elements, zinc and gallium, are consistent with either a FIP or volatility fractionation of the source material. Three other elements; sodium, copper, and germanium, show depletions relative to elements of similar first ionization potential which could be consistent with a volatility dependent fractionation model of the GCR source material.

The uncertainty in the sodium source abundance is dominated by the systematic uncertainty in the correction made for the secondary contribution to the observed abundance. The source values for the rare heavy elements beyond nickel are all limited by uncertainties in the measurements and in the solar system abundances. Even with the large CRIS geometry factor, collection rates for the trans-nickel nuclei are low, especially as solar maximum approaches. Nevertheless, a five-year total mission could allow a 50\% increase in the available data set. This, along with improvements in propagation models for elements from carbon to nickel, suggests that over the next few years, CRIS measurements do have the potential to discriminate between FIP and volatility as the controlling

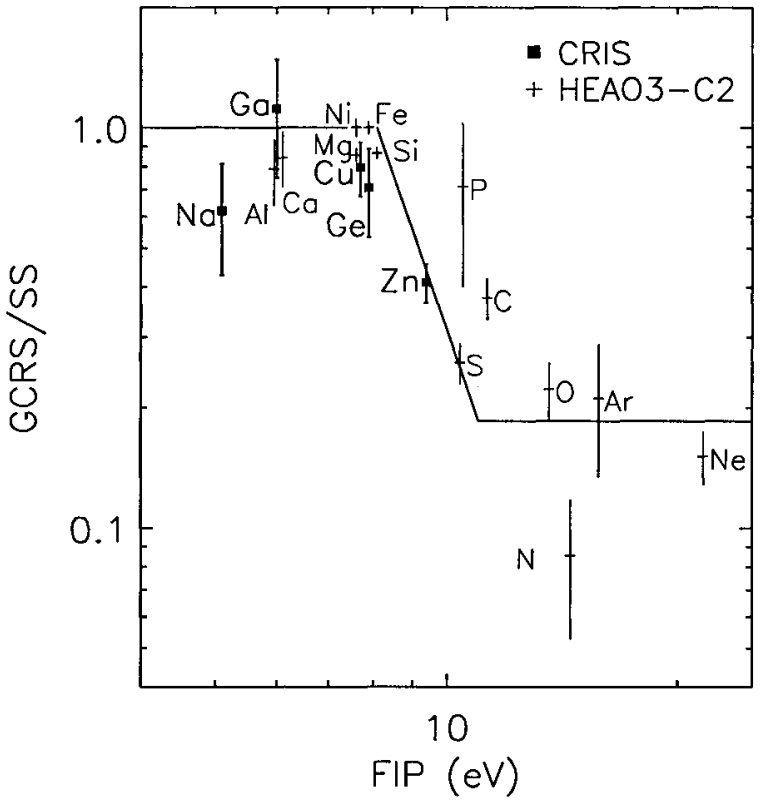

FIGURE 4. GCR Source / Solar System as a function of FIP ( $\mathrm{Fe} \equiv 1)$. HEAO data (13) added for context.

parameter for the elemental fractionation of the galactic cosmic ray source material.

This work was supported by NASA at the California Institute of Technology (under grant NAG5-6912), the Jet Propulsion Laboratory, the Goddard Space Flight Center, and Washington University.

\section{REFERENCES}

1. Stone, E.C., et al., Space Sci. Rev. 96, 285-356 (1998).

2. Geiss, J., Space Sci. Rev. 85, 241-252, (1998).

3. Hénoux, J.-C., Space Sci. Rev. 85, 215-226, (1998).

4. Cassé, M. \& Goret, P. ApJ 221, 703 (1978).

5. Meyer, J.-P., ApJS 57, 173-204 (1985).

6. Epstein, R.I., $M N R A S, 193,723$ (1980).

7. Bibring, J.-P., and Cesarsky,C.J. Proc. 17th ICRC (Paris), 2, 289 (1981).

8. Meyer, J.P., Drury, L., \& Ellison, D.C., ApJ 487, 182-196 (1997).

9. Anders, E. \& Grevesse, N., Geochim. Cosmochim. Acta 53, 197-214 (1989).

10. Tsao, C.H., Silberberg, R., Barghouty, A.F., Sihver, L., ApJ 451, 275 (1995).

11. Silberberg, R., Tsao, C.H., and Barghouty, A.F., ApJ 501, 911 (1998).

12. Tsao, C.H., Silberberg, R., and Barghouty, A.F., ApJ 501, 920 (1998).

13. Engelmann, J.J., et al., Astron. Astrophys. 233, 96-111 (1990).

14. Binns, W.R., et al., Proc. 18th ICRC (Bangalore) 9, 106 (1983).

15. Leske, R.A., ApJ 405, 567-583 (1993). 